\title{
Films de nitrure de carbone obtenus par ablation laser ArF du graphite en atmosphère d'azote. Corrélation entre l'émission du plasma et la composition du dépôt
}

\author{
C. Fuchs, R. Henck, E. Fogarassy, J. Hommet* et F. Le Normand* \\ CNRS, Laboratoire PHASE, UPR 292, BP. 20, 67037 Strasbourg cedex 2, France \\ * IPCMS-GSI, BP. 20, 67037 Strasbourg cedex 2, France
}

Résumé : L'analyse spectroscopique, de 110 à $600 \mathrm{~nm}$, de l'émission des plasmas créés lors de l'ablation par laser à excimères (ArF : $193 \mathrm{~nm}$ ) de cibles de graphite en atmosphère d'azote et sous vide, et l'analyse par spectroscopie de photoélectrons (XPS) des films $\mathrm{C}_{1-\mathrm{x}} \mathrm{N}_{\mathrm{x}}$ obtenus ont permis la mise en évidence d'une loi de corrélation entre la valeur de $x$ et la pression d'azote.

Le but de ce travail était la mise en évidence d'une éventuelle corrélation entre les spectres d'émission du plasma développé lors de l'ablation laser d'une cible de graphite sous faible atmosphère d'azote et la composition élémentaire des dépôts $\mathrm{C}_{1-\mathrm{x}} \mathrm{N}_{\mathrm{x}}$ obtenus. L'analyse spectroscopique de l'émission du plasma et l'obtention de dépôts ont été réalisées avec une chambre d'ablation conventionnelle équipée d'un monochromateur sous vide (McPherson 218, domaine d'analyse : 110 à $600 \mathrm{~nm}$ ). La cible tournante de graphite était irradiée sous incidence normale par un laser pulsé à excimères (ArF : $193 \mathrm{~nm}$, fluence : 6 $\mathrm{J} / \mathrm{cm}^{2}$ ). Les dépôts étaient recueillis sur un substrat placé à $5 \mathrm{~cm}$ de la cible et leur composition a été déterminée par spectroscopie de photoélectrons (XPS).

L'ablation de graphite sous vide ne donne lieu qu'à l'émission de huit raies atomiques du carbone situées entre 140 et $250 \mathrm{~nm}$ (les plus intenses sont observées à 165,70 et 247,86 nm) correspondant à des transitions radiatives des états excités les plus bas de l'atome vers l'état fondamental [1]. Lorsque l'ablation est effectuée en présence d'azote $(0,02$ à $10 \mathrm{mbar})$, les spectres d'émission sont rapidement dominés par la présence de 3 bandes du système violet du radical CN situées entre 330 et $420 \mathrm{~nm}$ (Fig. 1). Ces bandes apparaissent dès $0,05 \mathrm{mbar}$ et leur intensité augmente rapidement avec la pression d'azote pour atteindre un large maximum entre 0,5 et 0,8 mbar puis décroître lentement vers une valeur palier (Fig. 2, courbes en pointillés). La présence de cette forte émission laisse à penser que les radicaux $\mathrm{CN}$ sont formés au sein du plasma, probablement par chocs entre atomes excités de carbone et atomes d'azote provenant de la dissociation de la molécule. Cette hypothèse est corroborée par le fait que la variation, en fonction de la pression d'azote, de l'intensité des raies atomiques du carbone et de celle des bandes $\mathrm{CN}$ est décrite par des courbes similaires.

L'analyse XPS des dépôts obtenus pour des pressions d'azote égales à $0,1,0,5,1$ et 5 mbar et une fluence de $6 \mathrm{~J} / \mathrm{cm}^{2}$ a permis une estimation des proportions du nombre d'atomes libres de carbone et de ceux liés à l'azote, une fois $(\mathrm{C}-\mathrm{N})$ ou deux fois $(\mathrm{C}=\mathrm{N})$ (Fig. 2, traits pleins). La proportion de carbone lié $\mathrm{C}=\mathrm{N}$ crô̂t continuellement avec la pression d'azote, alors que celle de carbone lié $\mathrm{C}-\mathrm{N}$ passe par un maximum vers 1 mbar (comme l'intensité lumineuse des bandes $\mathrm{CN}$, courbes en pointillés). Inversement, la teneur en carbone libre passe par un minimum lorsque la pression avoisine 0,5 à $0,8 \mathrm{mbar}$. La similitude entre l'évolution des intensités lumineuses des bandes $\mathrm{CN}$ émises par le plasma et celle de la proportion de $\mathrm{C}-\mathrm{N}$ dans le dépôt est un argument supplémentaire en faveur de la création des espèces $\mathrm{CN}$ par chocs entre des atomes de carbone éjectés de la cible de graphite et ceux du gaz environnant lors de leur transit de la cible vers le substrat.

Sur une série d'échantillons obtenus sous 1 et 5 mbar d'azote, nous avons étudié la variation, avec la fluence du laser (de 4 à $18 \mathrm{~J} / \mathrm{cm}^{2}$ ), du taux d'incorporation d'azote dans les films déposés. Les mesures par XPS montrent que, pour des densités d'énergie proches de $5 \mathrm{~J} / \mathrm{cm}^{2}$, ce taux est de l'ordre de $10 \%$ alors que pour des fluences supérieures à $12 \mathrm{~J} / \mathrm{cm}^{2}$, la fraction totale d'azote incorporé est comprise entre 30 et $40 \%$ (Fig. 3). On voit que les composés formés correspondent, dans le meilleur des cas $(\mathrm{P}=1 \mathrm{mbar}$, fluence $\geq 12 \mathrm{~J} / \mathrm{cm}^{2}$ ), à un rapport molaire $\mathrm{N} / \mathrm{C}=0,4$ soit $\mathrm{x}=0,28$. 


\section{Références}

1. R. Henck, C. Fuchs, E. Fogarassy, J. Hommet, F. Le Normand, Mater. Res. Soc. Symp. Proc., Vol. 526 (1999).

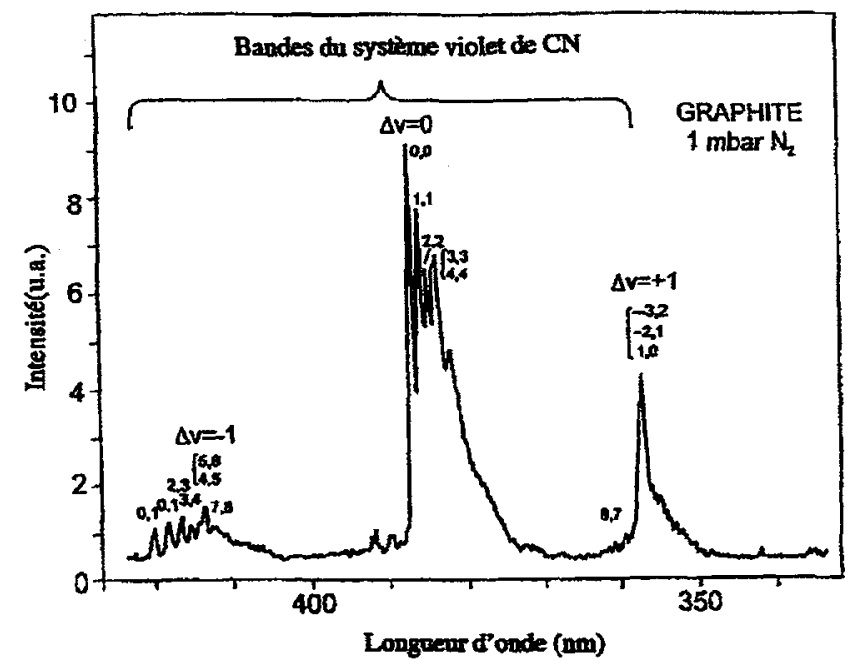

Figure 1 : Spectre d'Émission du plasma observe lors de l'ablation laser (AfF, $193 \mathrm{~nm}, 6 \mathrm{~J} / \mathrm{cm}^{2}$ ) de graphre dans 1 mbar d'azote.

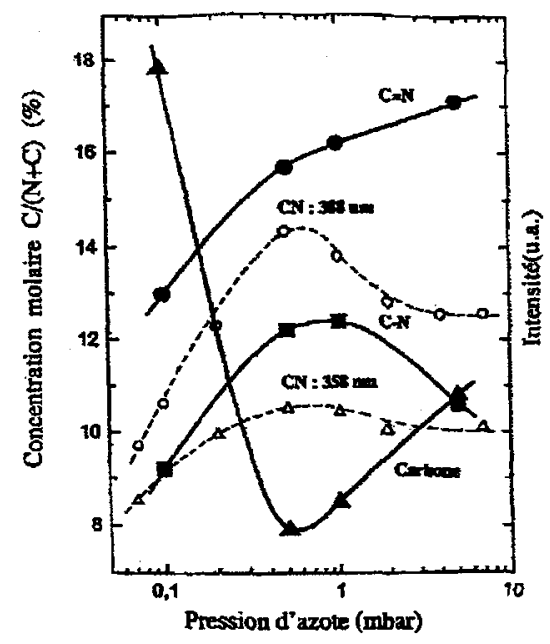

Figure 2: Variation de h concentration mobire en carbone libre et lié une fois ou deux (traits pleins) et de l'intensité lumineuse des bandes CN (en pointillés) en fonction de la pression d'azote.

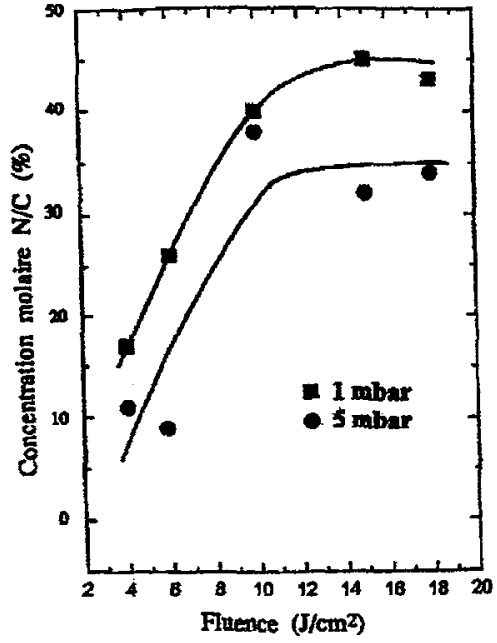

Figare 3 : Effet de la fluence du laser sur la stonc ture des dfpots $\mathrm{C}_{1-\mathrm{x}} \mathrm{N}_{\mathrm{X}}$ obtenus a I et 5 mbar d'azote. 